\title{
CUTANEOUS NECROTISING VASCULITIS - THERAPEUTIC FACT-A CASE REPORT
}

Dr Kiran. D. R, Dr Palaniswamy

1. Associate Professor, Department of General Medicine, Karuna Medical College, Vilayodi, Chittur, Palghat, Kerala.

2. Professor, Department of General Medicine, Karuna Medical College, Vilayodi, Chittur, Palghat, Kerala.

\section{CORRESPONDING AUTHOR:}

Dr. Kiran D R.,

Associate Professor,

Department of Medicine,

Karuna Medical College, Vilayodi,

Chittur, Palghat, Kerala.

Email id- kirandr_07@yahoo.co.in

ABSTRACT: INTRODUCTION: Mixed connective tissue disorder, unlike other connective tissue disorders have a milder course. MTCD with only necrotizing cutaneous vasculitis without organ damage respond well to Immunosuppresents and Steroids. CASE REPORT: Middle aged Young lady presented with multiple non healing large pressure sores and multiple nonblanchable purpuric lesions. She was bedridden, anaemic and with significant weight loss. All her major organ functions were normal. Her $\mathrm{U}_{1}$ RNP Antibody is positive and Skin Biopsy showed positive direct fluorescent test for IgG. She responded well to immunosuppresants and steroids. CONCLUSION: This patient who presented with MTCD, with predominant necrotizing cutaneous vasculitis and without major organ involvement showed good recovery and responded well to cyclophosphamide pulse therapy, daily azathioprine and good wound care.

KEY WORDS: Necrotising Vasculitis, MCTD, $U_{1}$ RNP Antibody, Immunosuppresants

\section{INTRODUCTION:}

Vasculitis is not a disease but rather a disease process (from Merck manual).Here we came across a MCTD with predominant necrotizing vasculitis involving only skin .This is been reported so as to stress the fact that MTCD , unlike other connective tissue disorders has milder course and necrotizing vasculitis confined to skin do well with immunosuppresives, steroids and good wound care 5 .

\section{CASE REPORT:}

Female patient aged 34 year old, presented with weight loss( more than 10\%), lethargy and multiple non healing open ulcers associated with necrotic base over pressure bearing areas predominantly confined to extensor areas of limbs of one year duration. She had intermittent, moderate grade fever without cough and rashes. She was bed ridden due to sever nonhealing disabling ulcers over the pressure bearing areas and joint contractures.

On examination, Patient is thin built and poorly nourished.

Multiple non blanchable purpuric lesions, multiple depigmented lesions on face and loss of scalp hair.

Pallor present, Febrile, Tachycardia present.

Systemic examination- Normal. 
Investigations: $\mathrm{Hb}$ 6gm/dl, WBC -8200, Eosinophils increased, Platelet count- 80,000, ESR-110, CRP elevated, LFT and RENAL FUNCTION: Normal

Urine Routine: Normal, No albumin or microscopic Haematuria

ECG: WNL

Echo: No pericardial fluid, EF - 60\%, Normal Function.

PFT: Normal curve, No features of interstitial pattern.

Chest X Ray: CP angles free and normal, lung parenchyma normal, No Infiltrates.

USG Abdomen: NO Organomegaly, pelvis normal.

Stool examination: No occult blood.

CT brain: Normal study.

NCS: Normal.

Ophthalmological Examination: Visual Aquity-6/6, Fundus -Normal.

ANA positive, RA Factor negative

ANCA negative, $\mathrm{U}_{1}$ RNP Antibody positive,

Skin Biopsy - direct fluorescent test positive for IgG, Complements were normal ,

HIV 1\&2 negative, Hepatitis B \& C Negative.

Diagnosis of MCTD was made with above findings.

\section{TREATMENT GIVEN:}

Patient was started with steroids, monthly pulse therapy of Cyclophosphamide and daily $2 \mathrm{mg}$ of Azathioprine. Other general conditions maintained with blood transfusion and supportive care. Wounds were been taken care of by debridement and allowed them to go for healing by secondary intention. Some of the wounds healed in one setting, but others required 2-3 attempts. Finally in six months duration all wounds healed well and now patient is ambulatory, self dependant with only Azathioprine daily.

\section{ACKNOWLEDGEMENT:}

I take this opportunity to extend my sincere thanks and indebtedness to all those persons and dignitaries who helped me to complete this work.

It gives pleasure to express my sense of gratitude to my professor Dr Palaniswamy for his guidance, encouragement and constant source of inspiration during case management. Above all I thank the Almighty for the successful completion of this work.

\section{DISCUSSION:}

MCTD also known as Sharp's syndrome an undifferentiated connective tissue disease. MCTD a combined feature of scleroderma, myositis, SLE and Rheumatoid Arthritis (with some source adding polymyositis, dermatomyositis and inclusion body myositis) and is thus considered as overlap syndrome. MCTD commonly causes joint swelling, malaise, Raynaud's phenomenon, Sjogren's syndrome, muscle inflammation and sclerodactyly. Distinguishing lab characteristics are positive speckled ANA and an anti $U_{1}$ RNP antibody. It is associated with HLA DR-41.

Vasculitis induced injury to blood vessel may lead to increased vascular permeability, vessel weakening that cause aneurysm formation, haemorrhage and intimal proliferation, thrombosis that result in obstruction and local ischemia ${ }^{2}$. It is critical to distinguish vasculitis occurring as a primary autoimmune disorder from vasculitis secondary to infection, drugs, malignancy or connective tissue disease such as SLE/ RA. Much of the diagnostic work up in a 
patient with suspected vasculitis is directed at excluding secondary causes that can mimic vasculitis ${ }^{3}$.

Immunoglobulin finding may be helpful in diagnosing MTCD. Indirect Immuno fluroscent test - Presence high titre of IgG against $U_{1} R N P$ as the only autoantibody support MTCD. The fluroscent auto nuclear antibody test typically reveals a speckled pattern of staining on HEP-2 substrate. Recent studies revealed that antibody directed to an appropriate specific epitop on $70 \mathrm{kd}$ are specifically associated with MTCD than other anti $70 \mathrm{k}$ antibody. Direct Immuno fluroscent test- Performed on lesional skin of patients with MTCD, this may reveal epidermal nuclear IgG staining. The staining is thought to be related to high titres $\mathrm{U}_{1} \mathrm{RNP}$ antibody in the patient seen. In some cases Lupus Band test may be positive (linear deposition of Ig, Fibrin and/or compliment components present at the basement membrane) ${ }^{4}$.

Treatment option includes corticosteroids, Immunosuppressive drugs to reduce the inflammation. Cyclophosphamide may be in severe vasculitis. Dramatic remission seen in patient with alternate day corticosteroid treatment with continuation of cyclophosphamide. Later corticosteroid was discontinued. Mean duration of remission was 22 months. No patient showed recurrence of disease during treatment with cytotoxic agents ${ }^{5}$. IV cyclophosphamide is better than oral and recommended at least six months. Substitution of Azathioprine after remission with cyclophosphamide did not increase the rate of relapse.

Newer treatment approach includes deoxysperagualin, achieved a high rate of disease remission and permitted prednisolone reduction. Other newer Immunosuppresives are Leflunamide, TNF antagonists - Infliximab and ENBREL. Colchicine not that much effective in skin va sculitis, but some showed improvement. Development of plasma exchange including semi specific immunoabsorption with L-tryptophan or Protein A columns to remove ANCA without depletion of non Ig plasma proteins and appear of comparable efficacy to plasma exchange ${ }^{6}$.

\section{CONCLUSION:}

MCTD, although called as overlap syndrome, here we came across MCTD without major organ involvement, without associated other connective tissue diseases. Predominant presentation was confined to skin as necrotizing cutaneous vasculitis. The disease here showed a chronic disabling course which provided enough time to treat with Pulse cyclophosphamide therapy, daily Azathioprine and good wound care. MCTD without predominant organ dysfunction and confined to skin manifestation as a good prognosis with regular treatment than the systemic vasculitis or MCTD with other connective diseases, which has mixed results to the regular treatment.

\section{REFERENCES:}

1 Aringer M, Steiner G, Smolen JS (Aug-03). "Does MCTD exist? Yes.” Rheumatic disease clinical North America. 31(3): 411-29.

2 Mandell BF, Hoffman G.S. Differrentiating the Vasculitis. Rheumatic disease clinical North America (1994); 20:409-42.

3 Hunder G. Vasculitis Diagnosis and therapy. Am. J Med: 1996; 100(22):375-455

4 H Ihn, K Yamane, N Yazawa, M Kuba, M Fujimoto, S Sato, K Kikuchi and K Tamaki; Distribution of antigen specificity of Anti $U_{1} R N P$ antibody in patient with systemic sclerosis. Clinical Experimental Immunology(1999)117(2);383-387.

5 FauciAS,Katz P,Haynes BF, Wolf SM:Cyclophosphamide therapy for sever necrotizing vasculitis(1979) vol 301:235- 238.

Journal of Evolution of Medical and Dental Sciences/Volume 1/Issue 3/July- Sept 2012 Page 104 
6 Jayne D (2000)Evidence based treatment of systemic vasculitis. Rheumatology(Oxford) 39:585-595.

\section{ABBREVIATIONS:}

MCTD: Mixed Connective Tissue Disease

HLA: Human Leucocyte Antigen

SLE: Systemic Lupus Erythematosis

RA: Rheumatoid Arthritis

RNP: Ribosomal Neucleo Protein

ANA: Anti Nuclear Antibody

ANCA: Anti Nuclear Cytoplasmic Antibody

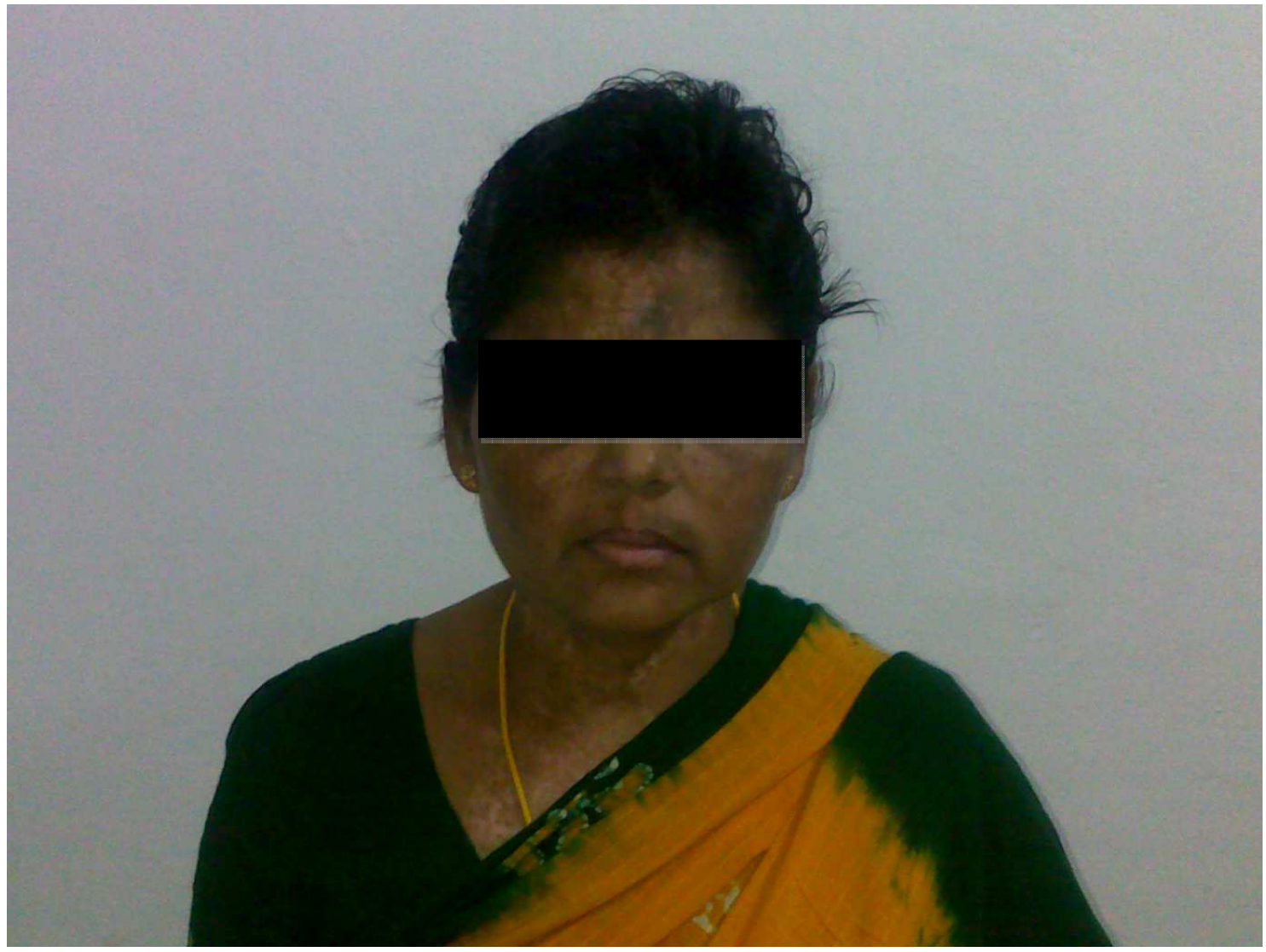

Post Treatment- Healed cutaneous Vasculitis on face, Post Healed Hypopigmented Lesion 